\title{
Defective Inflammatory Monocyte Development in IRF8-Deficient Mice Abrogates Migration to the West Nile Virus-Infected Brain
}

\author{
Rachael L. Terry ${ }^{a, b, d} \quad$ Celine Deffrasnes $^{a, b} \quad$ Daniel R. Getts $^{d}$ Carsten Minten $^{b, c}$ \\ Caryn van Vreden ${ }^{a, b}$ Thomas M. Ashhurst ${ }^{a, b}$ Meghann T. Getts ${ }^{d}$ \\ Rui Dan Vicki Xie ${ }^{\text {b, c }}$ lain L. Campbell ${ }^{\text {b, c }}$ Nicholas J.C. King ${ }^{\text {a, b }}$ \\ aDepartment of Pathology, bosch Institute and Marie Bashir Institute for Infectious Diseases and Biosecurity, \\ School of Medical Sciences, Sydney Medical School and 'School of Molecular Bioscience, Sydney University, \\ Sydney, N.S.W., Australia; ${ }^{\text {d} D e p a r t m e n t ~ o f ~ M i c r o b i o l o g y-I m m u n o l o g y, ~ N o r t h w e s t e r n ~ U n i v e r s i t y, ~ C h i c a g o, ~ I I I ., ~ U S A ~}$
}

\section{Key Words}

CCR2 - Encephalitis · Inflammatory monocytes · IRF8 ·

Ly6C+ monocytes $\cdot$ VLA-4 $\cdot$ West Nile virus

\begin{abstract}
IRF8 (interferon-regulatory factor-8) plays a critical role in regulating myeloid cell differentiation. However, the role of this transcription factor in the development of Ly6C+ inflammatory monocytes and their migration to the infected brain has not been examined. We have previously shown that West Nile virus (WNV) infection of wild-type (WT) mice triggers a significant increase in numbers of Ly6C+ monocytes in the bone marrow. These cells traffic via the blood to the infected brain, where they give rise to proinflammatory macrophages. Here, we show that WNV-infected IRF8-deficient (IRF8-/-) mice had significantly reduced numbers of Ly6C+ monocytes in the periphery, with few of these cells found in the blood. Furthermore, low numbers of inflammatory monocyte-derived macrophages were observed in the brains of IRF8-/- mice throughout infection. Adoptive transfer of IRF8-/- Ly6C+ monocytes demonstrated that these cells were intrinsically unable to traffic to the inflamed brain. Low expression of the chemokine receptor CCR2 and integrin VLA-4 by IRF8-/- monocytes likely contributed to this defect, as the interactions between these proteins and their li-
\end{abstract}

gands are critical for monocyte egress and migration to inflammatory foci. These data highlight a critical role for IRF8 in inflammatory monocyte differentiation and migration during WNV infection.

(c) 2014 S. Karger AG, Basel

\section{Introduction}

Inflammatory monocytes, identified in mice by high expression of Ly6C and the chemokine receptor CCR2, are recruited to most organs in response to infection or tissue damage, including the central nervous system (CNS), where they differentiate into proinflammatory macrophage and dendritic cell (DC) subsets [1-7]. On the other hand, Ly6C- $\mathrm{CX}_{3} \mathrm{CR}_{1}+$ monocytes are thought to be important for patrolling vessels and serve as a source of tissue macrophages and DC during homeostasis [8]. Although these phenotypically and functionally distinct subsets are well described, the developmental relationship between Ly6C+ and Ly6C- monocytes remains unclear $[9,10]$. Furthermore, the molecular pathways that control the development of these populations from their common progenitors are not well defined [11], but are of increasing interest. For example, Hanna et al. [12] have recently shown that the transcription factor NR4A1 
(Nur77) is critical for the differentiation of Ly6C- monocytes from their Ly6C+ precursors. Understanding these pathways is clearly an important step in resolving the dichotomy between Ly6C+ and Ly6C- monocytes, as well as for informing therapeutic interventions that target monocytes in inflammation, autoimmunity and myeloid leukemia [1-7].

Recent studies have examined the role of the interferon$\gamma$-stimulated transcription factor IRF8 (interferon regulatory factor-8) in monocyte development. Also known as ICSBP (interferon consensus sequence-binding protein), IRF8 is critical for the normal development of myeloid lineage cells. Hambleton et al. [13] recently showed that a patient with several loss-of-function mutations in the IRF8 gene completely lacked circulating monocytes and DC. In mice, IRF8 is upregulated during the differentiation of common myeloid progenitors and granulocytemacrophage progenitors from hematopoietic stem cells and promotes monocyte development at the expense of neutrophils [14, 15]. IRF8-deficient (IRF8-/-) mice show significant accumulation of granulocyte-macrophage progenitors and neutrophil expansion [16-18]. Also, IRF8-/- monocytes exhibit enhanced proliferation, resistance to apoptosis and defective differentiation into macrophage and DC subsets [17-21]. Recently, Becker et al. [18] reported that IRF8-/- mice lacked a significant population of Ly6C+ inflammatory monocytes in the blood. Kurotaki et al. [22] also showed that IRF8 is critical for Ly6C+ monocyte development, with reduced numbers of these cells in the blood, bone marrow (BM) and spleen of IRF8-/- mice. However, neither of these studies has addressed the role of IRF8 in monocyte development or migration during infection.

In this study, we directly examined the role of IRF8 in the development and migration of Ly6C+ monocytes under homeostatic and inflammatory conditions. We show that IRF8-/- mice have significantly reduced inflammatory monocyte precursors in the $\mathrm{BM}$, with the recently described splenic reservoir [23-25] also markedly diminished. Furthermore, these cells failed to traffic in high numbers to the West Nile virus (WNV)-infected brain. Adoptive transfer of equal numbers of BM-derived wildtype (WT) and IRF8-/- monocytes confirmed that IRF8-/- monocytes were intrinsically unable to migrate to the inflamed CNS. The inability to migrate was potentially the result of low CCR2 expression on IRF8-/monocytes, the chemokine receptor pivotal for Ly6C+ monocyte recruitment to sites of inflammation $[3,26-$ 28]. Furthermore, IRF8-/- inflammatory monocytes failed to significantly increase expression of activated
VLA-4 (very late antigen-4), the adhesion molecule critical for the migration of Ly6C+ monocytes to the WNVinfected CNS [6]. Together this work highlights the existence of a dynamic role for IRF8 in the differentiation of inflammatory monocytes, including the expression of key chemokine receptors and activation of adhesion molecules needed for cellular migration.

\section{Materials and Methods}

\section{Mice and Infection}

The Animal Ethics Committee of Sydney University approved all animal procedures (L02/1-2011/3/5466). WT or IRF8-/- mice [17] were used in this study. C57BL/6-7.2fms-EGFP mice [29] were intercrossed with C57BL/6 WT or IRF8-/- mice [17] to generate animals with eGFP+ myeloid lineage cells, which were used as donors in adoptive transfer studies. Mice were housed in HEPA filter-top cages under class II biohazard conditions, with food and water provided ad libitum. Female 8 -week-old mice were infected intranasally with $6 \times 10^{4}$ plaque forming units (PFU) WNV (Sarafend), as described previously $[3,30]$.

\section{Plaque Assays}

Plaque assays were performed as described previously $[3,30]$. Briefly, 10-fold dilutions of brain homogenates were used to infect confluent baby hamster kidney cells for $1 \mathrm{~h}$. Wells were overlaid with $1.5 \%(\mathrm{w} / \mathrm{v})$ low-gelling agarose II (Amresco) in $2 \times$ minimum essential medium (Gibco). Cells were incubated for 3 days and then fixed with $10 \%$ formalin (Fronine) for $2 \mathrm{~h}$ prior to plug removal. A 3\% crystal violet (Hopkins and Williams) dye solution in $20 \%$ methanol (Fronine) was used to stain fixed cells. Plaques were counted and the final PFU per gram/tissue was determined.

\section{Leukocyte Isolation and Flow Cytometry}

Leukocytes were isolated from BM, blood, spleens and brains on day (D) 0 to D7 post-infection (p.i.) and processed for flow cytometry, as described previously $[3,30]$. Antibodies against CD45, CD11b, Ly6C, Ly6G, CD115, F4/80, CD11c, MHC-II, CD16/32, CD3 and NK1.1 were obtained from Biolegend. Antibody against CD29 (VLA-4 $\beta 1$ chain activation epitope, clone 9EG7) was purchased from BD Biosciences and CCR2 from R\&D Systems. Samples were acquired on the FACS LSR-II (BD Biosciences) and analyzed using FlowJo (TreeStar).

\section{Ly6C Blockade}

To block Ly6C function in vivo, WNV-infected mice were injected with $100 \mu \mathrm{g}$ i.v. of purified anti-Ly6C antibody (clone $1 \mathrm{G} 7$. G10; Miltenyi Biotec) or rat IgG2a isotype control on D5 and D6 p.i. Brains were isolated on D7 p.i. and processed for flow cytometry.

\section{Adoptive Transfer}

For adoptive transfer studies, Ly6C+ WT and IRF8-/- monocytes were isolated and sorted from the BM, as described previously [3]. Cells were labeled with PKH26 (Sigma) and $2.0 \times 10^{6}$ cells were injected intravenously into mock-infected and WNV- 


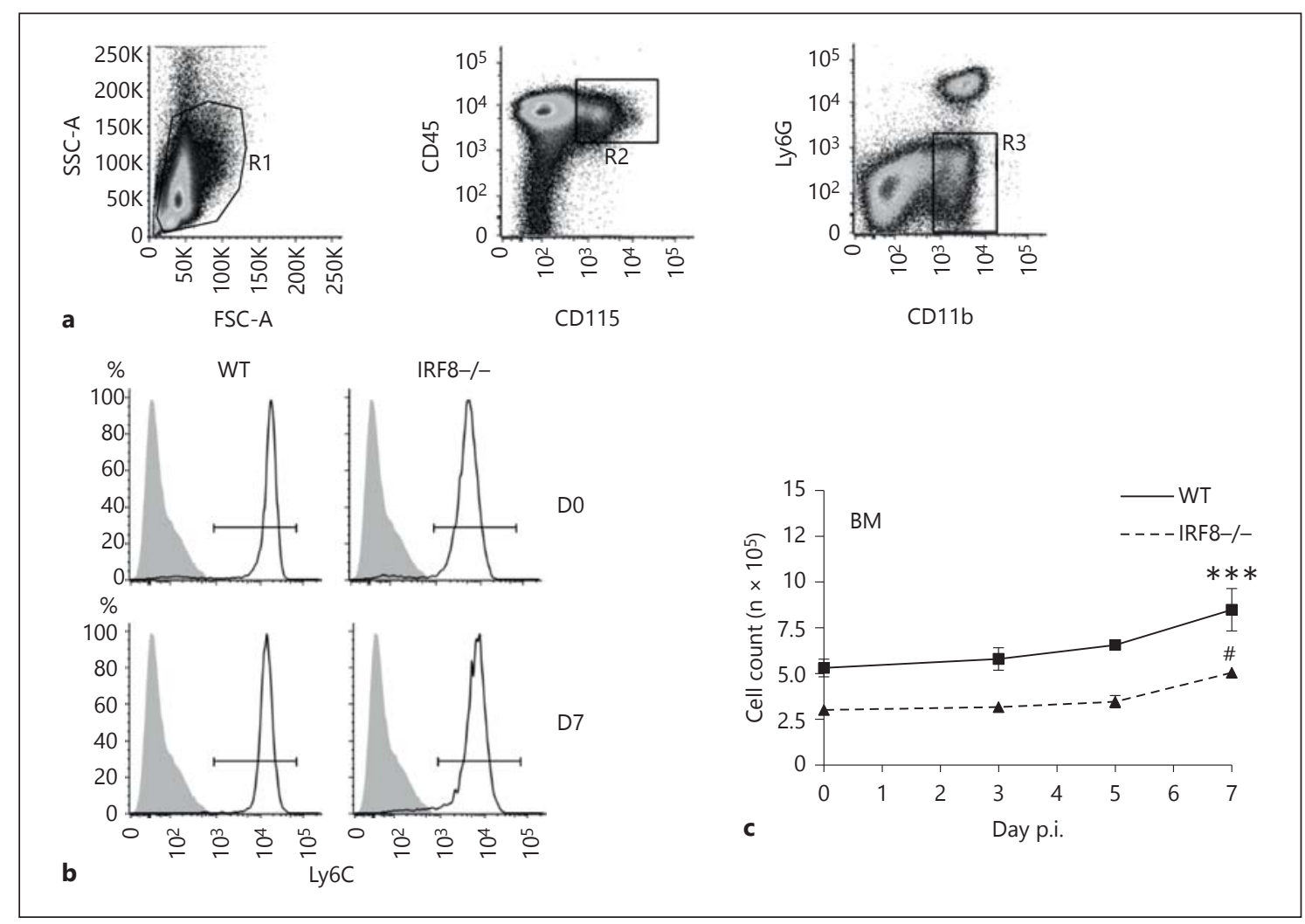

Fig. 1. IRF8-/- mice have significantly lower numbers of Ly6C+ monocytes in peripheral organs. BM, spleen and blood from D0, D3, D5 and D7 WNV-infected WT and IRF8-/- mice were processed for flow-cytometric analysis. Inflammatory monocytes were gated as CD 45+ CD115+ CD11b+ Ly6G- Ly6C+ cells (a) in the BM (b), spleen $(\mathbf{d})$ and blood $(\mathbf{f})$. Total numbers of Ly6C+ monocytes in the BM (c), spleen (e) and blood (g) were calculated using flow-cytometric percentages and absolute live cells counts for each organ. Flow-cytometric data shown are means \pm SD and represent 3 sepa-

infected recipients on D6 p.i., as described previously [3]. Organs were isolated from recipients on D7 p.i., and leukocytes were isolated and processed for flow cytometry.

\section{Immunohistology}

Immunohistology was performed on $8 \mu \mathrm{M}$ frozen brain sections as described previously [3, 30]. Lectin (from Bandeiraea simplicifolia) was purchased from Sigma. Antibodies against intercellular adhesion molecule-1, vascular cell adhesion molecule (VCAM)-1, CD11b, CCL2, CD45 and Gr-1 (Ly6C and Ly6G) were purchased from Biolegend. The WNV anti-non-structural protein 1 was a kind gift of Roy Hall (University of Queensland). Images were acquired on a fluorescent microscope (BX-51; Olympus), using DP manager 2.2.1 software (Olympus).

\section{Multiplex ELISA}

Multiplex plate ELISA (Quansys Biosciences) was performed as previously described [3], using the Quansys Q-plex mouse cytokine screen IR 16-plex and visualized on the Li-Cor rate experiments with 4 mice/group. Statistical analysis was conducted using one-way ANOVA and the Tukey-Kramer post hoc test. *** $\mathrm{p} \leq 0.001$ comparing the numbers of WT and IRF8-/- Ly6C+ monocytes at all time points (not shown). ${ }^{* *} \mathrm{p} \leq 0.01,{ }^{* * *} \mathrm{p} \leq 0.001$ comparing numbers of WT Ly6C+monocytes in the BM, spleen and blood on D7 p.i. to all other days. ${ }^{*} \mathrm{p} \leq 0.05$ comparing numbers of WT Ly6C+monocytes in the spleen on D5 p.i. to all other days. ${ }^{\#} \mathrm{p} \leq$ 0.05 IRF8-/- Ly6C+ monocytes on D7 p.i. to all other days.

(For figure 1d-g see next page.)

Odyssey IR Imaging System (Li-Cor Biotechnology). Images were analyzed using Quansys Q-view software (Quansys Biosciences).

\section{Statistics}

Graphs were made using Prism (GraphPad), with statistical analyses performed using InStat (GraphPad). An unpaired twotailed Student's t test or one-way ANOVA with a Tukey-Kramer post hoc test was performed. $\mathrm{p} \leq 0.05$ was considered significant (* $\left.\mathrm{p} \leq 0.05,{ }^{* *} \mathrm{p} \leq 0.005,{ }^{* * *} \mathrm{p} \leq 0.001\right)$.

\section{Results}

IRF8-/- Mice Lack Circulating Ly6C+ Monocytes

Our previous studies have shown that WNV infection of WT mice promotes Ly6C+ inflammatory monocyte 


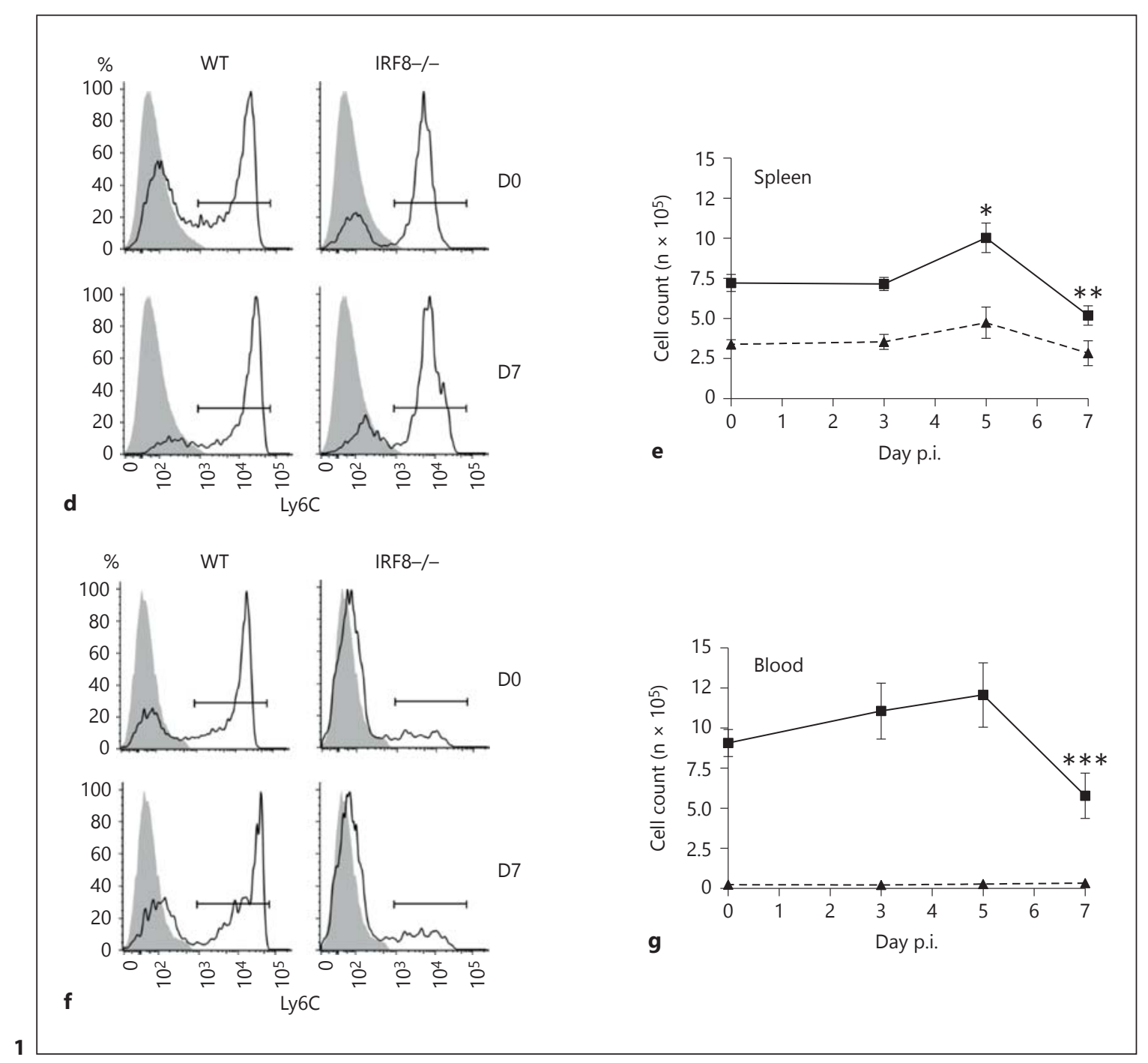

hematopoiesis in the $\mathrm{BM}$, egress into the circulation and recruitment to the CNS $[3,6]$. To determine if IRF8 is critical for the development and migration of these cells under homeostatic and inflammatory conditions, we utilized a well-characterized and robust model of WNV encephalitis $[3,6]$. Following infection with $6 \times 10^{4} \mathrm{PFU}$, both WT and IRF8-/- mice succumbed by D7 p.i. with similar disease kinetics. At a 10 -fold lower virus dose, i.e. $6 \times 10^{3} \mathrm{PFU}, 50 \%$ of WT mice survived infection with sterilizing immunity, while all IRF8-/- succumbed to disease. Terminal viral titers in WT and IRF8-/- brains were similar following high- and low-dose infection. Strikingly, very few infiltrating myeloid cells were observed at either dose in the brains of IRF8-/- mice at these time points (online suppl. fig. 1A-C; for all online suppl. material, see www.karger.com/doi/10.1159/000365972).
To investigate why IRF8-/- mice showed a significant decrease in the infiltration of these cells into the inflamed CNS, the BM, blood, spleens and brains of WNV-infected WT and IRF8-/- mice were processed for flow cytometry at D0, D3, D5 and D7 p.i., and inflammatory monocytes were gated as CD45+ CD11b+CD115+ Ly6GLy6C+ cells (fig. 1a). There were no significant differences in the expression of CD11b, CD115, F4/80, CD62L, Ly6G or CD11c by WT and IRF8-/- Ly6C+ monocytes in the BM, spleen, or blood (online suppl. fig. 2A). However, in the BM, only $50 \%$ of the numbers of Ly6C+ inflammatory monocytes were observed in IRF8-/- mice compared to the WT at all time points throughout infection (fig. 1b, c). Similar to previous observations, WNV infection significantly increased the numbers of WT Ly6C+ monocytes in the BM on D7 p.i. 
[3]; this increase was greater than that observed in the BM of IRF8-/- animals (fig. 1b, c).

In the spleen, there were also half the numbers of Ly6C+ monocytes in IRF8-/- mice compared to the WT at all time points investigated (fig. $1 \mathrm{~d}, \mathrm{e}$ ). WNV infection resulted in a significant increase in numbers of WT Ly6C+ monocytes in the spleen on D5 p.i., with a significant decrease observed by $\mathrm{D} 7 \mathrm{p}$.i. In comparison, numbers of Ly6C+ monocytes in the IRF8-/- spleen did not significantly change throughout the course of infection (fig. 1d, e). Compared to the BM and spleen, in the blood very few circulating Ly6C+ monocytes were observed in IRF8-/animals compared to the WT at all time points (fig. 1f, g). On D7 p.i., similar to the spleen, significantly reduced numbers of circulating Ly6C+ monocytes were observed in the blood of WT mice (fig. 1f, g). Interestingly, in the BM, blood and spleen, IRF8-/- Ly6C+ monocytes expressed significantly lower levels of Ly6C than WT monocytes (fig. 1b, d, f; online suppl. fig. 2B-D).

\section{IRF8-/- Ly6C+ Monocytes Are Intrinsically Unable to} Traffic to the Virus-Infected Brain

WNV infection of WT mice recruits large numbers of inflammatory monocytes to the brain from D5 p.i., which give rise to CD45+ CD115+ CD11b+ Ly6G- Ly6C+ inflammatory macrophage populations (fig. 2a, b) [6]. As expected, large numbers of Ly6C+ macrophages were observed in the WT WNV-infected brain from D5 to D7 p.i. In alignment with our histological findings (online suppl. fig. 1C), very few of these cells were observed in the WNVinfected IRF8-/- brain, although at D7 p.i. there was a significant increase compared to all other days investigated (fig. 2b, c). These findings were supported by immunohistology, in which only a small myeloid infiltrate could be observed in the WNV-infected IRF8-/- brain relative to the WT on $\mathrm{D} 7$ p.i. (fig. $2 \mathrm{~d}-\mathrm{o}$ ). Significantly reduced expression of Ly6C by IRF8-/- inflammatory monocytes in the periphery likely contributed to im-

Fig. 2. IRF8-/- mice show significantly reduced Ly6C+ macrophage infiltration into the WNV-infected brain. Brains from D0, D3, D5 and D7 WNV-infected WT and IRF8-/- mice were processed for flow-cytometric analysis. CD45+ CD115+ CD11b+ Ly6G- Ly6C+ inflammatory monocyte-derived macrophages were gated in the brain $(\mathbf{a}, \mathbf{b})$. Total Ly6C+ macrophage numbers were calculated using flow-cytometric percentages and absolute live cells counts for each brain (c). Immunohistology was also performed on brain sections from D0 and D7 WNV-infected WT and IRF8-/- mice on D7 p.i. Sections were stained with CD45 (red; $\mathbf{d}-\mathbf{g}), \mathrm{CD} 11 \mathrm{~b}$ (red; h-k) or Ly6C cells ( $\mathrm{red} ; \mathbf{I - o}$ ) and counterstained paired trafficking to the CNS (fig. 1b, d, f; online suppl. fig. 2B-D). Intravenous injection of WT mice with Ly6Cblocking antibody on D5 and D6 p.i. reduced the numbers of inflammatory monocytes in the D7 p.i. WNV-infected brain by approximately $66 \%$ (online suppl. fig. $2 \mathrm{E}$ ).

The regulation of monocyte migration occurs both at the site of inflammation and within the BM $[3,26]$. To test whether impaired recruitment of IRF8-/- monocytes was a result of the inability to exit the BM effectively, eGFP+ (CD115+) Ly6C+ WT or IRF8-/- monocytes were sorted from the BM (fig. 3a). These cells were also labeled with the membrane dye PKH26 to confirm the identity of the transferred cells and to make certain that eGFP (CD115) expression was neither lost nor downregulated after transfer. $2.0 \times 10^{6} \mathrm{WT}$ or IRF8-/- monocytes were transferred into matched mock-infected or WNVinfected recipients on D6 p.i. Substantial numbers of transferred Ly6C+ WT monocytes trafficked into the WNV-infected WT brain on D7 p.i. and gave rise to Ly6C+ macrophages (fig. 3b, c). However, the number of transferred Ly6C+ WT monocytes recruited to the WNVinfected IRF8-/- brain on D7 p.i. was only two thirds of that recruited to the WT brain. In comparison, low numbers of transferred Ly6C+IRF8-/- monocytes were found in the D7 WT or IRF8-/- infected brain, although there was a very small increase in recruitment to the WT brain (fig. 3b, c). These data indicate that impaired migration of IRF8-/- inflammatory monocytes to the CNS is principally due to a monocyte-intrinsic defect, but additional factors may also contribute to reduced recruitment in IRF8-/- mice, such as the reduced proinflammatory environment generated in the WNV-infected IRF8-/-CNS compared to the WT (online suppl. fig. 3A-F). Consistent with reduced trafficking of IRF8-/- Ly6C+ monocytes to the CNS, there was significant accumulation of these cells in the BM and spleen of WNV-infected WT and IRF8-/recipients following transfer, compared to transferred WT Ly6C+ monocytes (fig. 3d, e).

with DAPI (blue) and lectin (green). Flow-cytometric data shown are means \pm SD and represent 3 separate experiments with 4 mice/ group. Statistical analysis was conducted using one-way ANOVA and the Tukey-Kramer post hoc test. ${ }^{* * *} \mathrm{p} \leq 0.001$ comparing the numbers of WT and IRF8-/- Ly6C+ macrophages on D5 and D7 (not shown). ${ }^{* *} \mathrm{p} \leq 0.001$ in comparing the numbers of WT Ly6C+ macrophages on D5 or D7 p.i. to all other days. ${ }^{\#} \mathrm{p} \leq 0.05$ comparing the numbers of IRF8-/- Ly6C+ macrophages on D7 p.i. to all other days. Immunohistology was performed on 3 entire sagittal brain sections from a minimum of 3 mice/group, conducted twice.

(For figure see next page.) 


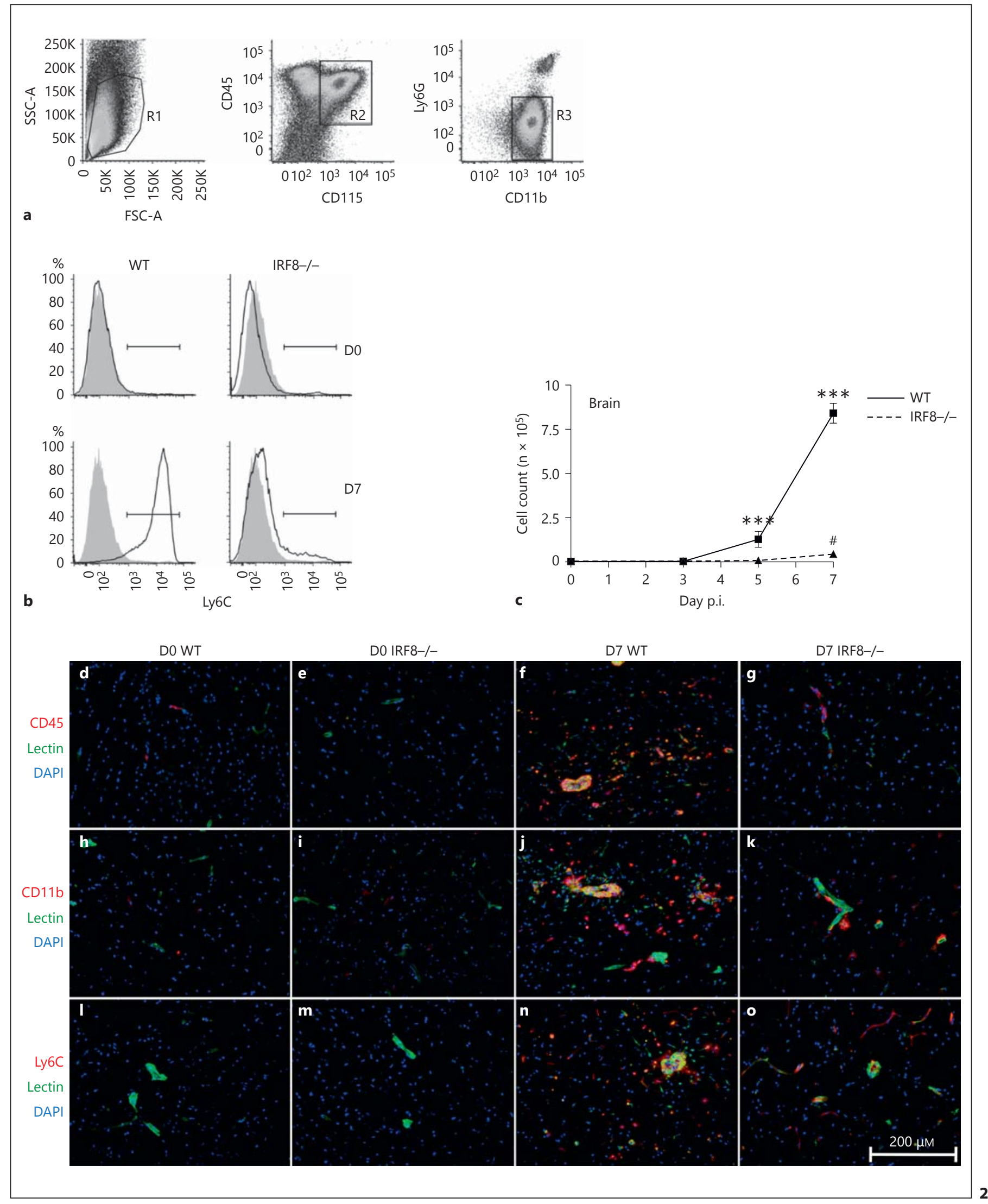




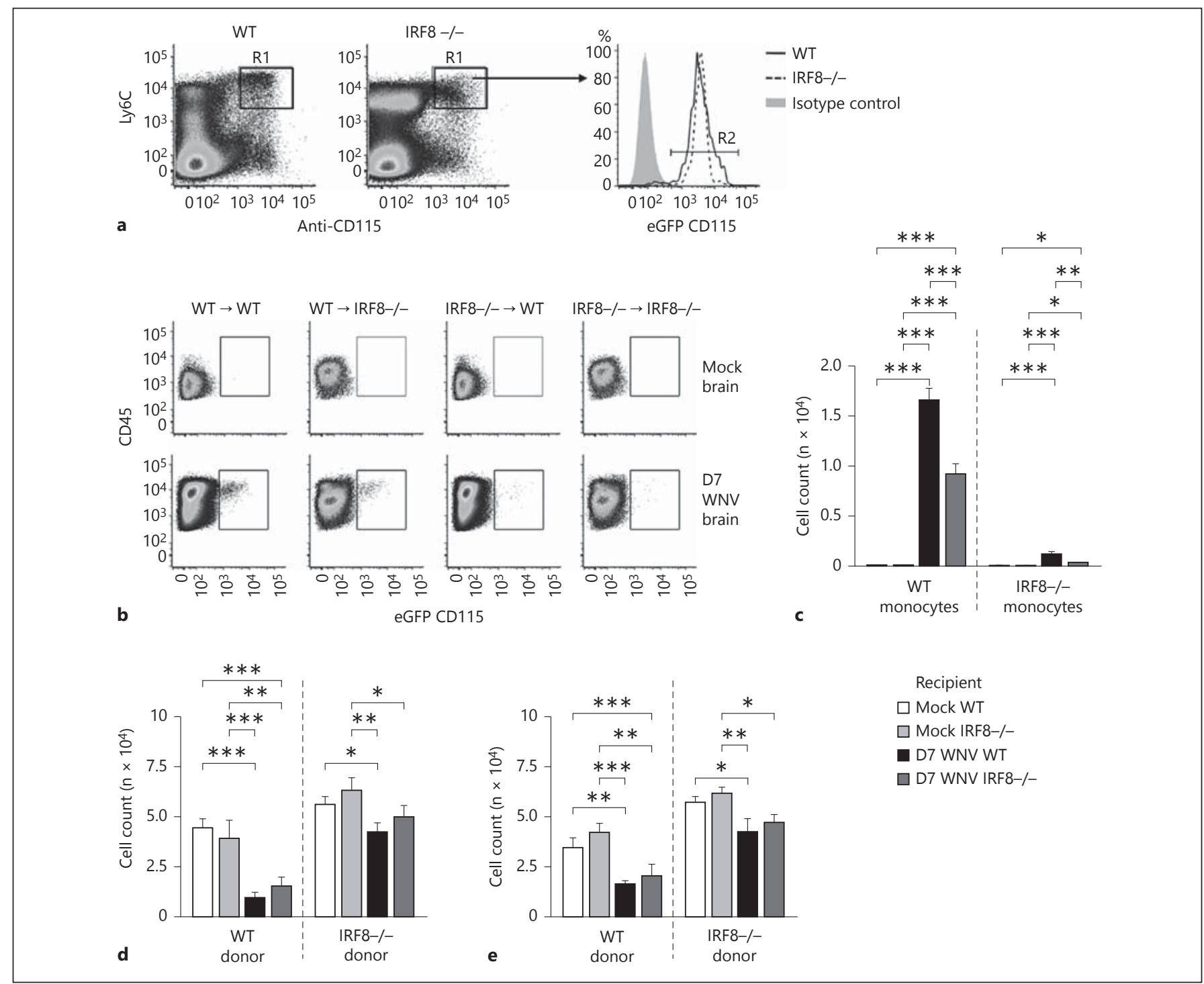

Fig. 3. IRF8-/- monocytes are intrinsically unable to migrate to the WNV-infected brain. C57BL/6-7.2fms-EGFP mice [29] were intercrossed with C57BL/6 WT or IRF8-/- mice [17] to generate animals with eGFP+ myeloid lineage cells, which were used as donors in adoptive transfer studies. Flow cytometry was used to confirm that BM-derived CD45+ CD115+ CD11b+ Ly6G- Ly6C+ monocytes were GFP+ (a). BM-derived GFP+ Ly6C+ monocytes from mock-infected and WNV-infected WT and IRF8-/- donors were sorted on D6 p.i. and labeled with PKH26. $2.0 \times 10^{6}$ cells were injected intravenously into mock-infected or WNV-infected WT

\section{IRF8-/- Ly6C+ Monocytes Express Low Levels of CCR2}

To investigate why IRF8-/- monocytes showed impaired migration to the infected brain, the CCL2/CCR2 chemokine/chemokine receptor axis was investigated. CCL2, produced by WNV-infected neurons, is critical or IRF8-/- recipients on D6 p.i. On D7 p.i., the brains, BM and spleens of recipient animals were processed for flow-cytometric analysis and transferred GFP+ PKH26+ cells were gated (b). Total numbers of transferred Ly6C+ WT and IRF8-/- monocytes in the brain (b, c), BM (d) and spleen (e) were calculated using flowcytometric percentages and absolute live cells counts for each organ. Flow-cytometric data shown are means \pm SD and represent 3 separate experiments with 4 mice/group. Statistical analysis was conducted using one-way ANOVA and the Tukey-Kramer post hoc test. ${ }^{*} \mathrm{p} \leq 0.05,{ }^{* *} \mathrm{p} \leq 0.01,{ }^{* * *} \mathrm{p} \leq 0.001$.

for the egress of Ly6C+monocytes from the BM and recruitment from the circulation into the brain [3]. CCL2 was produced at similar levels in D7 WNV-infected WT and IRF8-/- brains (fig. 4a-q). In comparison, IRF8-/Ly6C+ monocytes expressed significantly lower levels of the CCL2 receptor, CCR2, than their WT counterparts 


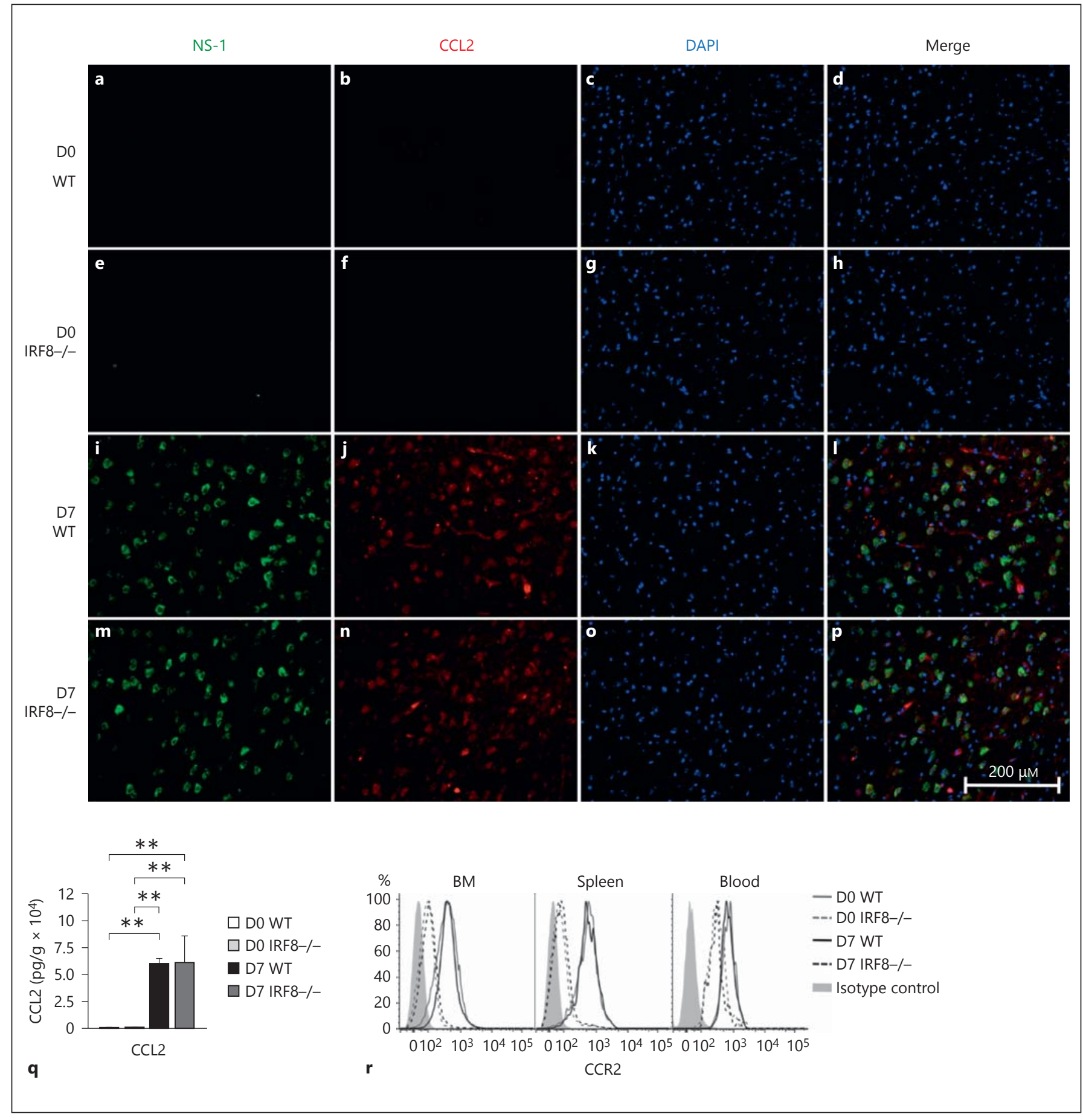

Fig. 4. IRF8-/- monocytes express low levels of the chemokine receptor CCR2. Brains from D0 and D7 WNV-infected WT and IRF8-/- mice were collected and processed into sections for fluorescent immunohistology (a-p). Sections were stained with WNV NS-1 (green), CCL2 (red) and counterstained with DAPI (blue). Brains from D0 and D7 WNV-infected WT and IRF8-/- mice were also collected and processed for CCL2 ELISA (q). BM, blood and spleens of D0 and D7 WNV-infected WT and IRF8-/- mice were also isolated and processed for flow-cytometric analysis.
CD45+ CD115+ CD11b + Ly6G- Ly6C+ monocytes were gated and expression of the chemokine receptor CCR2 was compared (r). Flow-cytometric data shown are means \pm SD and represent 3 separate experiments with 4 mice/group. Statistical analysis was conducted using one-way ANOVA and the Tukey-Kramer post hoc test. ${ }^{* *} \mathrm{p} \leq 0.01$. Immunohistology was performed on 3 entire sagittal brain sections from a minimum of 3 mice/group, conducted twice. CCL2 ELISA data shown are means \pm SD and were performed twice with a minimum of 4 mice/group. 


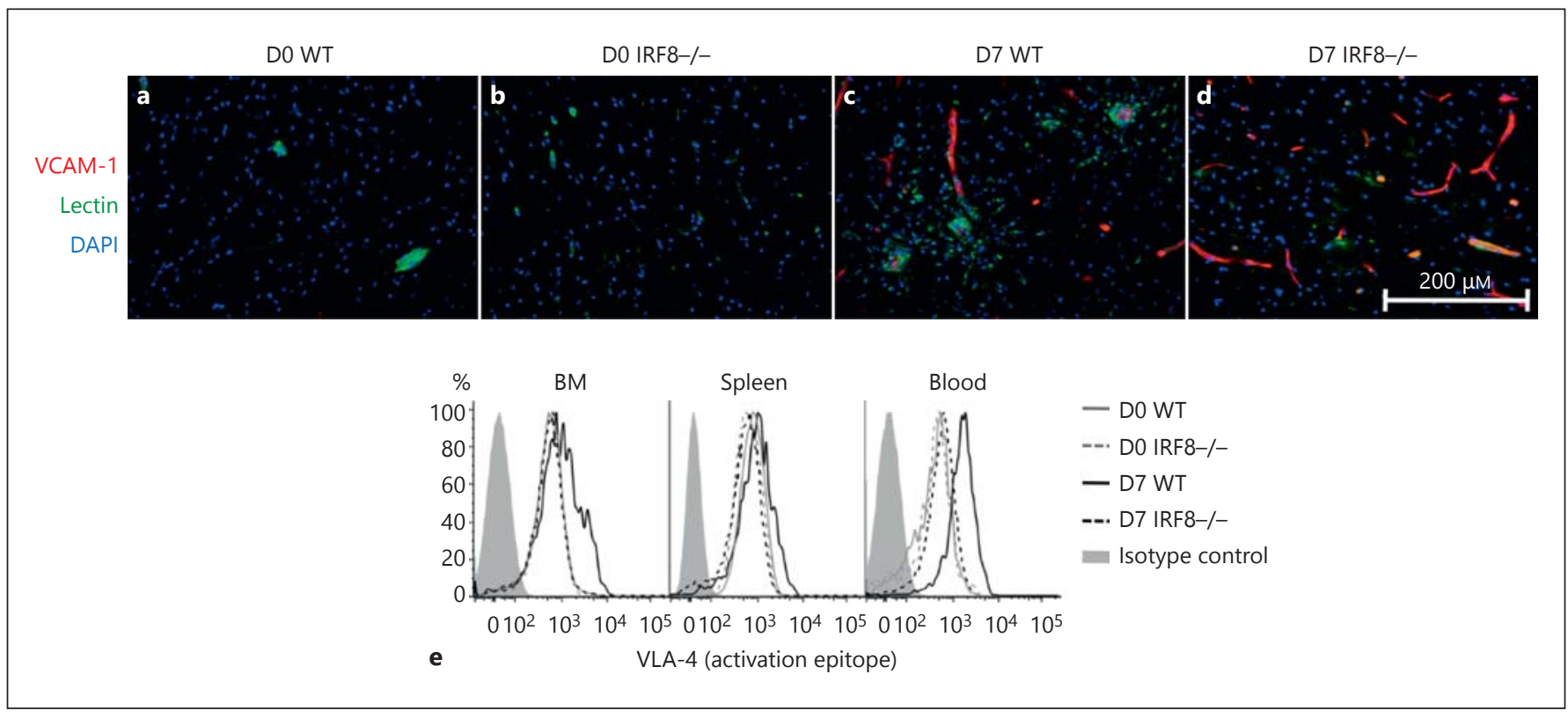

Fig. 5. IRF8-/- monocytes express low levels of the integrin VLA4. Mock-infected and WNV-infected WT and IRF8-/- brain sections were processed for immunohistology and stained for VCAM1 (red; a-d). Slides were also stained with DAPI (blue) and lectin (green). The BM, blood and spleens of mock-infected and WNVinfected WT and IRF8-/- mice were processed for flow-cytomet-

in the BM, blood and spleen (fig. 4r; online suppl. fig. 4AC). Studies using CCR2-/- or CCL2-neutralized mice highlight the importance of the CCL/CCR2 axis for Ly6C+ monocyte migration. In these mice, Ly6C+ monocytes accumulate in the BM during homeostasis and infection, unable to traffic to sites of inflammation $[3,26-$ 28]. Thus, low levels of CCR 2 expression by Ly6C+ IRF8-/- monocytes likely contributed to their inability to accumulate in the blood or traffic to the WNV-infected brain.

Next, adhesion molecule expression was examined. Ly6C+ monocyte migration to the CNS is dependent on the expression of the integrin VLA-4, which binds to the adhesion molecule VCAM-1, expressed by endothelium perfusing the inflamed brain [6]. We found that VCAM1 expression was upregulated to similar levels in the D7 WT and IRF8-/- WNV-infected brain (fig. 4a-d). To determine if the expression of activated VLA- 4 differed between WT and IRF8-/- inflammatory monocytes, we stained these cells with an antibody that recognizes an activation epitope of this integrin. While WNV infection significantly upregulated the expression of active VLA-4 on WT monocytes in the BM, spleen and blood, IRF8-/cells failed to increase expression of this key integrin mol- ric analysis on D7 p.i. Ly6C+ monocytes were gated and the expression levels of the activated integrin VLA-4 (e) were compared. Immunohistology was performed on 3 entire sagittal brain sections from a minimum of 3 mice/group, conducted twice. Flowcytometric data shown are representative of 3 separate experiments with 4 mice/group.

ecule to a similar extent, particularly in the blood (fig. 5e; online suppl. fig. 4D-F). These data indicate that impaired migration of IRF8-/- monocytes is also likely contributed to by an inability to sufficiently activate VLA-4 in response to inflammatory stimuli.

\section{Discussion}

In this study, we investigated the role of the transcription factor, IRF8, in the development and migration of Ly6C+ monocytes during homeostasis and WNV infection of the CNS. Our data indicate that IRF8 is important for the development of Ly6C+ inflammatory monocytes. IRF8-/- mice showed a 50\% reduction in the numbers of these cells in the BM, as well as the spleen, which has recently been identified as a hematopoietic source of undifferentiated monocytes [23-25]. These data are consistent with recent studies which show that IRF8 plays an important role in the differentiation of both Ly6C+ and Ly6Cmonocytes $[18,22]$. Importantly, the lack of IRF8 was associated with increased mortality following WNV infection, consistent with previous findings that macrophages are important in virus clearance [31]. 
Although CNS infection increased relative numbers of both WT and IRF8-/- Ly6C+ monocytes in the BM, infection was not sufficient to overcome the inherent defect in monocyte differentiation in IRF8-/- mice, as it did not increase numbers of Ly6C+ monocytes to levels similar to those of the WT. Strikingly, there was almost a complete lack of circulating Ly6C+ monocytes in IRF8-/mice. These data indicate that IRF8 expression may also be important for the emigration of Ly6C+ monocytes from the $\mathrm{BM}$ and spleen into the blood.

CCL2/CCR2 interactions are critical for enhancing firm adhesion between the integrin VLA-4 and its ligands $[32,33]$. Thus, we hypothesized that low expression of CCR 2 by Ly6C+ IRF8-/- monocytes compared to the WT likely resulted in reduced VLA-4 activation, thus hampering diapedesis. Accordingly, we found that IRF8-/- monocytes in the periphery failed to significantly upregulate expression of active VLA-4 in response to CNS infection to the same extent as WT cells. Furthermore, cross-linking of Ly6C is important for the activation of LFA- 1 and VLA-4 on CD 8 T cells and promotes the homing of these cells to the lymph nodes [3436]. We and others have also shown that Ly6C is critical for the migration of inflammatory monocytes to the inflamed CNS [37]. Based on our findings and the current literature [34-36], we speculate that high expression of Ly6C by inflammatory monocytes is important for the migration of these cells into the infected brain, most likely by enhancing the interactions of VLA-4 with VCAM-1, expressed on the brain endothelium, since the interaction of these molecules is crucial for monocyte infiltration into the brain. Thus, lower expression of Ly6C by IRF8-/- inflammatory monocytes may also have contributed to impaired infiltration of the WNVinfected brain.

In summary, IRF8 is critical for normal Ly6C+ monocyte development. IRF8-/- Ly6C+ monocytes are unable to accumulate in the circulation and fail to traffic to the infected brain. We propose that low expression of Ly6C, CCR 2 and VLA -4 by these cells contributed to this intrinsic defect, as these proteins are critical for the migration of Ly6C+ monocytes to the WNV-infected brain.

\section{Acknowledgments}

We thank the members of the Sydney University animal houses and the Histopathology Laboratory for technical support. This work was supported by the National Health and Research Council (NHMRC APP1007757 and NHMRC 512413). R.L.T. was the recipient of an Australian Postgraduate Award and Wenkart Foundation Scholarship during this study.

\section{Disclosure Statement}

The authors have no conflict of interest to disclose.

\section{References}

1 King IL, Dickendesher TL, Segal BM: Circulating Ly-6C+ myeloid precursors migrate to the CNS and play a pathogenic role during autoimmune demyelinating disease. Blood 2009;113:3190-3197.

2 Bao Y, Kim E, Bhosle S, Mehta H, Cho S: A role for spleen monocytes in post-ischemic brain inflammation and injury. J Neuroinflammation 2010;7:92.

- 3 Getts DR, Terry RL, Getts MT, Muller M, Rana S, Shrestha B, Radford J, Van Rooijen N, Campbell IL, King NJ: Ly6C+ 'inflammatory monocytes' are microglial precursors recruited in a pathogenic manner in West Nile virus encephalitis. J Exp Med 2008;205:23192337.

4 Mildner A, Schlevogt B, Kierdorf K, Bottcher C, Erny D, Kummer MP, Quinn M, Bruck W, Bechmann I, Heneka MT, Priller J, Prinz M: Distinct and non-redundant roles of microglia and myeloid subsets in mouse models of Alzheimer's disease. J Neurosci 2011;31: 11159-11171.

IRF8-Deficient Inflammatory Monocytes Do Not Traffic to the Virus-Infected Brain
5 Mildner A, Mack M, Schmidt H, Bruck W, Djukic M, Zabel MD, Hille A, Priller J, Prinz M: CCR2+Ly-6Chi monocytes are crucial for the effector phase of autoimmunity in the central nervous system. Brain 2009;132: 2487-2500.

6 6 Getts DR, Terry RL, Getts MT, Muller M, Rana S, Deffrasnes C, Ashhurst TM, Radford J, Hofer M, Thomas S, Campbell IL, King NJ: Targeted blockade in lethal West Nile virus encephalitis indicates a crucial role for very late antigen (VLA)-4-dependent recruitment of nitric oxide-producing macrophages. J Neuroinflammation 2012;9:246.

-7 Getts DR, Terry RL, Getts MT, Deffrasnes C, Muller M, van Vreden C, Ashhurst TM, Chami B, McCarthy D, Wu H, Ma J, Martin A, Shae LD, Witting P, Kansas GS, Kuhn J, Hafezi W, Campbell IL, Reilly D, Say J, Brown L, White MY, Cordwell SJ, Chadban SJ, Thorp EB, Bao S, Miller SD, King NJ: Therapeutic inflammatory monocyte modulation using immune-modifying microparticles. Sci Transl Med 2014;6:219ra217.
8 Geissmann F, Auffray C, Palframan R, Wirrig C, Ciocca A, Campisi L, Narni-Mancinelli E, Lauvau G: Blood monocytes: distinct subsets, how they relate to dendritic cells, and their possible roles in the regulation of T-cell responses. Immunol Cell Biol 2008;86:398-408.

>9 Geissmann F, Manz MG, Jung S, Sieweke MH, Merad M, Ley K: Development of monocytes, macrophages, and dendritic cells. Science 2010;327:656-661.

10 Ley K, Miller YI, Hedrick CC: Monocyte and macrophage dynamics during atherogenesis. Arterioscler Thromb Vasc Biol 2011;31: 1506-1516.

11 Auffray C, Sieweke MH, Geissmann F: Blood monocytes: development, heterogeneity, and relationship with dendritic cells. Annu Rev Immunol 2009;27:669-692.

12 Hanna RN, Carlin LM, Hubbeling HG, Nackiewicz D, Green AM, Punt JA, Geissmann F, Hedrick CC: The transcription factor NR4A1 (Nur77) controls bone marrow differentiation and the survival of Ly6C(-) monocytes. Nat Immunol 2011;12:778-785. 


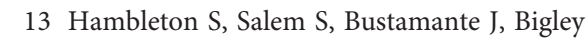
V, Boisson-Dupuis S, Azevedo J, Fortin A, Haniffa M, Ceron-Gutierrez L, Bacon CM, Menon G, Trouillet C, McDonald D, Carey P, Ginhoux F, Alsina L, Zumwalt TJ, Kong XF, Kumararatne D, Butler K, Hubeau M, Feinberg J, Al-Muhsen S, Cant A, Abel L, Chaussabel D, Doffinger R, Talesnik E, Grumach A, Duarte A, Abarca K, Moraes-Vasconcelos D, Burk D, Berghuis A, Geissmann F, Collin M, Casanova JL, Gros P: IRF8 mutations and human dendritic-cell immunodeficiency. N Engl J Med 2011;365:127-138.

14 Wang H, Lee CH, Qi C, Tailor P, Feng J, Abbasi S, Atsumi T, Morse HC 3rd: IRF8 regulates B-cell lineage specification, commitment, and differentiation. Blood 2008;112: 4028-4038.

15 de Bruin AM, Libregts SF, Valkhof M, Boon L, Touw IP, Nolte MA: IFNgamma induces monopoiesis and inhibits neutrophil development during inflammation. Blood 2012;119: 1543-1554.

-16 Scheller M, Foerster J, Heyworth CM, Waring JF, Lohler J, Gilmore GL, Shadduck RK, Dexter TM, Horak I: Altered development and cytokine responses of myeloid progenitors in the absence of transcription factor, interferon consensus sequence binding protein. Blood 1999;94:3764-3771.

-17 Holtschke T, Lohler J, Kanno Y, Fehr T, Giese N, Rosenbauer F, Lou J, Knobeloch KP, Gabriele L, Waring JF, Bachmann MF, Zinkernagel RM, Morse HC 3rd, Ozato K, Horak I: Immunodeficiency and chronic myelogenous leukemia-like syndrome in mice with a targeted mutation of the ICSBP gene. Cell 1996;87:307-317.

18 Becker AM, Michael DG, Satpathy AT, Sciammas R, Singh H, Bhattacharya D: IRF-8 extinguishes neutrophil production and promotes dendritic cell lineage commitment in both myeloid and lymphoid mouse progenitors. Blood 2012;119:2003-2012.

19 Edelson BT, Kc W, Juang R, Kohyama M, Benoit LA, Klekotka PA, Moon C, Albring JC, Ise W, Michael DG, Bhattacharya D, Stappenbeck TS, Holtzman MJ, Sung SS, Murphy TL, Hildner K, Murphy KM: Peripheral CD103+ dendritic cells form a unified subset developmentally related to CD8alpha+ conventional dendritic cells. J Exp Med 2010;207:823-836.

-20 Tsujimura H, Tamura T, Ozato K: Cutting edge: IFN consensus sequence binding protein/IFN regulatory factor 8 drives the development of type I IFN-producing plasmacytoid dendritic cells. J Immunol 2003;170: 1131-1135.
21 Baccala R, Gonzalez-Quintial R, Blasius AL, Rimann I, Ozato K, Kono DH, Beutler B, Theofilopoulos AN: Essential requirement for IRF8 and SLC15A4 implicates plasmacytoid dendritic cells in the pathogenesis of lupus. Proc Natl Acad Sci USA 2013;110:29402945.

22 Kurotaki D, Osato N, Nishiyama A, Yamamoto M, Ban T, Sato H, Nakabayashi J, Umehara M, Miyake N, Matsumoto N, Nakazawa M, Ozato K, Tamura T: Essential role of the IRF8-KLF4 transcription factor cascade in murine monocyte differentiation. Blood 2013;121:1839-1849.

23 Robbins CS, Chudnovskiy A, Rauch PJ, Figueiredo JL, Iwamoto Y, Gorbatov R, Etzrodt M, Weber GF, Ueno T, van Rooijen N, Mulligan-Kehoe MJ, Libby P, Nahrendorf M, Pittet MJ, Weissleder R, Swirski FK: Extramedullary hematopoiesis generates Ly- $6 \mathrm{C}^{\text {high }}$ monocytes that infiltrate atherosclerotic lesions. Circulation 2012;125:364-374.

24 Leuschner F, Rauch PJ, Ueno T, Gorbatov R, Marinelli B, Lee WW, Dutta P, Wei Y, Robbins C, Iwamoto Y, Sena B, Chudnovskiy A, Panizzi P, Keliher E, Higgins JM, Libby P, Moskowitz MA, Pittet MJ, Swirski FK, Weissleder R, Nahrendorf M: Rapid monocyte kinetics in acute myocardial infarction are sustained by extramedullary monocytopoiesis. J Exp Med 2012;209:123-137.

25 Swirski FK, Nahrendorf M, Etzrodt M, Wildgruber M, Cortez-Retamozo V, Panizzi P, Figueiredo JL, Kohler RH, Chudnovskiy A, Waterman P, Aikawa E, Mempel TR, Libby P, Weissleder R, Pittet MJ: Identification of splenic reservoir monocytes and their deployment to inflammatory sites. Science 2009;325: 612-616.

26 Serbina N, Pamer E: Monocyte emigration from bone marrow during bacterial infection requires signals mediated by chemokine receptor CCR2. Nat Immunol 2006;7:311-317.

27 Lim JK, Obara CJ, Rivollier A, Pletnev AG, Kelsall BL, Murphy PM: Chemokine receptor Ccr2 is critical for monocyte accumulation and survival in West Nile virus encephalitis. J Immunol 2011;186:471-478.
8 Davison AM, King NJ: Accelerated dendritic cell differentiation from migrating Ly6C(lo) bone marrow monocytes in early dermal West Nile virus infection. J Immunol 2011; 186:2382-2396.

29 Sasmono RT, Oceandy D, Pollard JW, Tong W, Pavli P, Wainwright BJ, Ostrowski MC, Himes SR, Hume DA: A macrophage colonystimulating factor receptor-green fluorescent protein transgene is expressed throughout the mononuclear phagocyte system of the mouse. Blood 2003;101:1155-1163.

30 Getts D, Matsumoto I, Muller M, Getts M, Radford J, Shrestha B, Campbell I, King N: Role of IFN-gamma in an experimental murine model of West Nile virus-induced seizures. J Neurochem 2007;103:1019-1030.

31 Ben-Nathan D, Huitinga I, Lustig S, van Rooijen N, Kobiler D: West Nile virus neuroinvasion and encephalitis induced by macrophage depletion in mice. Arch Virol 1996;141:459469 .

32 Imhof BA, Aurrand-Lions M: Adhesion mechanisms regulating the migration of monocytes. Nat Rev Immunol 2004;4:432444.

33 Weber C, Springer TA: Interaction of very late antigen- 4 with VCAM- 1 supports transendothelial chemotaxis of monocytes by facilitating lateral migration. J Immunol 1998;161: 6825-6834.

34 Hanninen A, Maksimow M, Alam C, Morgan DJ, Jalkanen S: Ly6C supports preferential homing of central memory CD8+ T cells into lymph nodes. Eur J Immunol 2011;41:634644.

35 Jaakkola I, Merinen M, Jalkanen S, Hanninen A: Ly6C induces clustering of LFA-1 (CD11a/ CD18) and is involved in subtype-specific adhesion of CD8 T cells. J Immunol 2003;170: 1283-1290.

36 Hanninen A, Jaakkola I, Salmi M, Simell O, Jalkanen S: Ly-6C regulates endothelial adhesion and homing of CD8(+) T cells by activating integrin-dependent adhesion pathways. Proc Natl Acad Sci USA 1997;94:68986903.

-37 Butovsky O, Siddiqui S, Gabriely G, Lanser AJ, Dake B, Murugaiyan G, Doykan CE, Wu PM, Gali RR, Iyer LK, Lawson R, Berry J, Krichevsky AM, Cudkowicz ME, Weiner HL: Modulating inflammatory monocytes with a unique microRNA gene signature ameliorates murine ALS. J Clin Invest 2012;122: 3063-3087. 